\title{
AIAA-2001-1335 NONLINEAR ANALYSIS AND SCALING LAWS FOR NONCIRCULAR COMPOSITE STRUCTURES SUBJECTED TO COMBINED LOADS
}

\author{
Mark W. Hilburger, ${ }^{*}$ Cheryl A. Rose, ${ }^{*}$ and James H. Starnes, Jr. ${ }^{+}$ \\ NASA Langley Research Center \\ Hampton, Virginia 23681-001
}

\begin{abstract}
$\underline{\text { Abstract }}$
Results from an analytical study of the response of a built-up, multi-cell noncircular composite structure subjected to combined internal pressure and mechanical loads are presented. Nondimensional parameters and scaling laws based on a first-order shear-deformation plate theory are derived for this noncircular composite structure. The scaling laws are used to design sub-scale structural models for predicting the structural response of a full-scale structure representative of a portion of a blended-wing-body transport aircraft. Because of the complexity of the full-scale structure, some of the similitude conditions are relaxed for the sub-scale structural models. Results from a systematic parametric study are used to determine the effects of relaxing selected similitude conditions on the sensitivity of the effectiveness of using the sub-scale structural model response characteristics for predicting the full-scale structure response characteristics.
\end{abstract}

\section{Introduction}

The increased performance requirements of future transport aircraft, and the projected increased demand for air travel, suggest that more efficient transport aircraft concepts are the needed. An example of a revolutionary concept for an efficient, large transport aircraft is a blended-wing-body (BWB) type of aircraft, which blends the wings and the fuselage into a single lifting surface. In order to satisfy the weight and performance requirements for the BWB aircraft and other advanced concepts, lightweight structures that exploit the high specific strength, the high specific stiffness, and the design flexibility of advanced composite materials offer a desirable design advantage over conventional metallic materials. The effective design of advanced structural concepts using composite materials requires a thorough understanding of the response and failure characteristics of these structures, and a thorough understanding of how these response and failure characteristics are influenced

\footnotetext{
Aerospace Engineer. Mechanics and Durability Branch. Member, AIAA

${ }^{\dagger}$ Senior Engineer, Structures and Materials Competency. Fellow, AIAA.

Copyright (1) 2001 by the American Instutute of Aeronautics and Astronautics, Inc. No copyright is asserted in the United States under Title 17, U.S. Code. The $U$. S. Government has a royalty-free license to exercise all rights under the copyright claimed herein for Governmental Purposes. All other rights are reserved by the copyright owner.
}

by changes in structural design parameters. Obtaining this understanding requires the development of new and advanced design and analysis methodologies that are appropriate for the structural concepts and material systems being studied, and the verification of these analysis methodologies through experiments. Furthermore, certification of any new design requires extensive structural testing, since databases and design experience may not exist for the structural concepts and advanced composite materials of interest. However, an experimental investigation of composite structural components is both costly and time consuming, particularly if the structural components are large. Consequently, scaling laws that can be used to relate test results of sub-scale structural models to the response and test results of full-scale structures could be of value. A series of less expensive tests of subscale structural models could potentially be used to complement a reduced set of tests of full-scale structures required for analysis verification and design certification. Reducing the required number of full-scale structural tests in this manner could significantly reduce the time and cost of the design development for a new concept.

Previous studies of the design of sub-scale structural models for predicting the response of full-scale shell structures have been conducted. ${ }^{1-3}$ In these studies, complete similitude and partial similitude between the sub-scale structural model and the full-scale structure are discussed. In order for there to be complete similitude between the sub-scale structural model and the full-scale structure, all of the scaling laws must be satisfied simultaneously. In the case of complete similitude, the responses of the sub-scale structural model and the fullscale structure are identical. In some cases, however, complete similitude is difficult to achieve. Such a difficulty may occur when a sub-scale structural model is developed to represent the behavior of a full-scale complex built-up structure subjected to complex loading conditions. Consequently, relaxation of some of the similitude conditions is required, and the sub-scale structural model and the full-scale structure are only partially similar. This relaxation of similitude conditions results in differences in the response characteristics of the sub-scale structural model and the full-scale structure, and intelligent selection of the appropriate sub-scale structural 
model is required if this model is to be used for predicting the desired response in the full-scale structure.

The present paper has two objectives. The first objective is to present the results of an analytical study of the behavior of a complex, noncircular multi-cell composite structure subjected to combined internal pressure and mechanical loads. These results were determined using the STAGS (STructural Analysis of General Shells) nonlinear finite element code. ${ }^{4}$ The second objective is to present the results of the derivation of nondimensional parameters from the governing equations of motion for selected components of the noncircular multicell composite structure of interest, and to use these parameters to develop scaling laws for designing sub-scale structural models that could be used for structural tests. Scaled models with both complete and partial similitude are discussed. In the case of partial similitude between the sub-scale structural model and the full-scale structure, results from analytical parametric studies are presented, and these results are used to determine the effects of relaxing selected similitude conditions or scaling laws on the sensitivity of the effectiveness of using the subscale structural model response characteristics for predicting the full-scale structure response characteristics.

Finite-element Models and Analysis

\section{Structural Models ...}

The geometry of the full-scale structure analyzed in the present study is representative of a portion of the center-body region of a blended-wing-body aircraft, and is defined in Fig. 1. This region is referred to herein as the center-body structure. The span-wise and chord-wise dimensions and spar depth of the full-scale center-body structure are equal to $185 \mathrm{in}, 477 \mathrm{in.}$, and $120 \mathrm{in.}$., respectively. The leading edge structure is represented by a cylindrical shell segment with a radius equal to $60 \mathrm{in}$. and an arc-length equal to $188.5 \mathrm{in}$. (180 of arc). The cover panels and leading-edge structures are thick sandwich structures with a 5.0-inch-thick aluminum honeycomb core and 0.110-inch-thick graphite-epoxy facesheets. The spars are thick sandwich structures with a 2.0 -inch- thick aluminum honeycomb core and 0.110-inch-thick graphite-epoxy facesheets. The graphite-epoxy facesheets are built up from graphite-fiber warp-knit preforms that are infused with an epoxy resin. Each preform is a stack of graphite-epoxy material that is equivalent to nine layers of unidirectional pre-impregnated graphiteepoxy tape material with $44.7,42.7$ and $12.6 \%$ of $0^{\circ}$, $45^{\circ}$, and $90^{\circ}$ fibers, respectively. Each stack has a cured thickness equal to 0.055 inches. The effective material properties for the graphite-epoxy preform material, and for the honeycomb core are provided in Tables 1 and 2, respectively. The $0^{\circ}$ fiber direction, and the core ribbon direction are aligned with the $x$-axis (span-wise direction) of the center-body structure shown in Fig. 1. The loading condition for the structure consists of an applied internal pressure, $p=15 \mathrm{psi}$, and an applied span-wise compressive load, $N_{x}=4500 \mathrm{lbf} / \mathrm{in}$.

Two 1/12-scale models, referred to herein as subscale structural models, of the full-scale center-body structure were analyzed in the present study. Total similitude is satisfied between the first sub-scale structural model and the full-scale center-body structure. In this case, the sub-scale structural model has the same material properties and sandwich construction as the full-scale structure. Furthermore, all dimensions of the sub-scale sandwich construction are $1 / 12$ of the full-scale sandwich structural dimensions. The second sub-scale structural model is made with a monolithic-laminate construction, and is only partially similar to the full-scale structure. In this model, the cover panels and leadingedge structure are 0.220 -inch-thick monolithic laminates, and the spars are 0.110-inch-thick monolithic laminates. The material properties given in Table 1 for the graphite-epoxy preform material are assumed to be representative of the monolithic laminates. Loads applied to the sub-scale structural models were obtained from the appropriate scaling laws for the loads, which are presented in a subsequent section.

A typical finite element model used to simulate the response of both the full-scale and the sub-scale structural models is shown in Fig. 1. The structure with the

Table 1. Engineering constants for the graphite-epoxy preform material

\begin{tabular}{cccc}
\hline \hline $\left.\mathbf{E}_{\mathbf{x},}, \mathbf{m s i}\right)$ & $\mathbf{E}_{\mathbf{y},}(\mathbf{m s i})$ & $\left.\mathbf{G}_{\mathbf{x y},}, \mathbf{m s i}\right)$ & $v_{\mathbf{x y}}$ \\
\hline 9.25 & 4.67 & 2.27 & 0.4 \\
\hline \hline
\end{tabular}

Table 2. Engineering constants for the aluminum honeycomb core

\begin{tabular}{cccccc}
\hline \hline $\left.\mathbf{E}_{\mathbf{x},}, \mathbf{k s i}\right)$ & $\left.\mathbf{E}_{\mathbf{y},}, \mathbf{k s i}\right)$ & $\mathbf{E}_{\mathbf{z},}(\mathbf{k s i})$ & $\mathbf{G}_{\mathbf{x y},}(\mathbf{k s i})$ & $\mathbf{G}_{\mathbf{x z},}(\mathbf{k s i})$ & $\mathbf{G}_{\mathbf{y z},}(\mathbf{k s i})$ \\
\hline 0.5 & 0.5 & 97 & 0.5 & 57 & 24 \\
\hline \hline
\end{tabular}


monolithic laminates was modeled using the standard STAGS 410 quadrilateral shell element in the STAGS element library. The STAGS 410 element is a flat facettype of element, and is based on Kirchoff-Love shell theory and the nonlinear Lagrangian strain tensor. Each of the shell element nodes has six degrees of freedom, including three translational degrees of freedom, $u, v$ and $w$, and three rotational degrees of freedom, $r u, r v$, and $r w$ about the $x, y$ and $z$ axes, respectively. The sandwich structures were modeled using the STAGS 840 sandwich element. The STAGS 840 sandwich element is composed of upper and lower facesheets that are joined or held apart by a generally anisotropic core layer. The facesheets of the sandwich element are standard STAGS 410 quadrilateral shell elements. The core of the sandwich element, defined as the region between the upper and lower face sheets, is assumed to have generally anisotropic three dimensional elastic properties. The kinematics and specific elastic energy of the core are derived in terms of the facesheet displacement shape functions.

Kinematic boundary conditions applied to the model shown in Fig. 1 consist of setting the $v$ and $w$ displacements of the cover panels equal to zero along the boundary corresponding to $y=0$ inches, and setting the $u$ displacement of the structure equal to zero on the boundary corresponding to $x=185$ inches. The loading condition for the model consists of two parts. The internal pressure is simulated by applying a uniform lateral pressure to the cover panels, the aft spar, and the leadingedge structure. In addition, a distributed span-wise tension load is applied to the center-body structure, along the $x=0$ inches boundary, to simulate a bulkhead pressure load. For the combined loading condition, the spanwise compression load is simulated by applying a distributed load along the $x=0$ edges of the center-body structure.

\section{Nonlinear Analysis Procedure}

The structural response characteristics were predicted analytically using the STAGS (STructural Analysis of General Shells) nonlinear shell analysis code. STAGS is a finite-element code developed for the nonlinear static and dynamic analysis of general shells, and includes the effects of geometric and material nonlinearities in the analysis. The code uses the modified and full Newton methods for its nonlinear solution algorithms, and accounts for large rotations in a shell by using a corotational algorithm at the element level. The Riks pseudo arc-length path-following method is used to continue a solution past the limit points of a nonlinear response.

The nonlinear response of the center-body structures subjected to combined internal pressure and span- wise compression loads was determined using the following analysis procedure. First, a live pressure load was applied to the structure, and then a span-wise compression load was applied to the pressurized structure.

\section{Nondimensional Parameters and Scaling Laws}

Nondimensional parameters and scaling laws governing the nonlinear response of shear-deformable, builtup noncircular composite structures subjected to combined internal pressure and mechanical loads are presented in this section. Nondimensional parameters for the cover panels and spars have been derived from a first-order shear-deformation plate theory. A nondimensionalization procedure, following the procedures developed by Stein ${ }^{5}$ and Nemeth, ${ }^{6}$ has been applied to the plate governing equations of motion, which produced a set of nondimensional parameters in terms of the plate geometric parameters and material stiffnesses. The basic premise of the procedure is to make the field variables and their derivatives of order one, to minimize the number of independent parameters required to characterize the structural behavior, and to avoid introducing a preferential direction into the nondimensional equations. The derivation of the nondimensional equations is given in Appendix A. Scaling laws for built-up noncircular composite structures subjected to combined loads are developed using the resulting nondimensional parameters. For completeness, similar nondimensional parameters and scaling laws could be developed for the curved leading-edge structure. However, it was found that the parameters and scaling laws developed for the cover panels were sufficient to characterize the response of the leading-edge structure for the geometry and loading conditions considered in this study. Therefore, additional nondimensional parameters and scaling laws for the curved leading-edge structure were not developed.

\section{Nondimensional Parameters}

The governing equations of motion for shear deformable plates based on first-order shear-deformation plate theory are given by Eqns. (A1) - (A5) in Appendix $A$, and the corresponding nondimensional parameters are presented in this section. The corresponding nondimensional membrane parameters are given by Eqs. (1) - (3) and the nondimensional bending parameters are given by Eqs. (4) - (8), as follows

$$
\begin{gathered}
\mu_{1}=\left(\frac{L_{2}}{L_{1}}\right)^{2} \sqrt{\frac{A_{11}}{A_{22}}} \\
\mu_{2}=\frac{A_{66}}{\sqrt{A_{11} A_{22}}}
\end{gathered}
$$




$$
\begin{aligned}
\mu_{3} & =\frac{A_{12}+A_{66}}{\sqrt{A_{11} A_{22}}} \\
\alpha_{1} & =\frac{L_{2}}{L_{1}} \sqrt{\frac{D_{11}}{D_{22}}} \\
\alpha_{2} & =\frac{L_{1}}{L_{2}} \frac{D_{66}}{\sqrt{D_{11} D_{22}}} \\
\alpha_{3} & =\frac{D_{12}+D_{66}}{\sqrt{D_{11} D_{22}}} \\
\alpha_{4} & =\frac{L_{1} L_{2} A_{55}}{\sqrt{D_{11} D_{22}}}
\end{aligned}
$$

and

$$
\alpha_{5}=\frac{1}{L_{1}}\left(\frac{\sqrt{A_{11} A_{22} D_{11} D_{22}}}{A_{11} A_{22}-A_{12}^{2}}\right)^{1 / 2}
$$

where $A_{11}, A_{12}, A_{22}$ and $A_{66}$ are the shell membrane stiffnesses and $D_{11}, D_{12}, D_{22}$ and $D_{66}$ are the shell bending stiffnesses found in classical laminated plate theory, and $L_{1}$ and $L_{2}$ represent the characteristic dimensions of a plate element. In the context of the center-body structural configuration, $L_{1}$ corresponds the span-wise dimension of a plate element and $L_{2}$ corresponds to the chord-wise dimension of a plate element. Parameters representing the ratios of transverse shear stiffnesses and the plate aspect ratio are defined in Eqs. (9) and (10), respectively, as follows

$$
\begin{gathered}
\Gamma=\frac{G_{13}}{G_{23}} \\
\Lambda=\frac{L_{1}}{L_{2}}
\end{gathered}
$$

where $G_{13}$ and $G_{23}$ are transverse shear stiffnesses of the plate. Nondimensional in-plane displacements of the reference surface are given by

$$
U=\frac{A_{11} A_{22}-A_{12}^{2}}{\sqrt{A_{11} A_{22} D_{11} D_{22}}} L_{1} u
$$

and

$$
V=\frac{A_{11} A_{22}-A_{12}^{2}}{\sqrt{A_{11} A_{22} D_{11} D_{22}}} L_{2} v
$$

and have a character that is similar to $u L_{1} / t^{2}$ and $v L_{2}$ ' $t^{2}$, respectively, where $t$ is the thickness of the plate. The nondimensional normal displacement is given by

$$
W=\left(\frac{A_{11} A_{22}-A_{12}^{2}}{\sqrt{A_{11} A_{22} D_{11} D_{22}}}\right)^{1 / 2} w
$$

and has a character that is similar to $w / t$. Nondimensional in-plane loading parameters are given by

$$
\begin{aligned}
& n_{x}=\frac{N_{x} L_{2}^{2}}{\sqrt{D_{11} D_{22}}} \\
& n_{x y}=\frac{N_{x y} L_{1} L_{2}}{\sqrt{D_{11} D_{22}}}
\end{aligned}
$$

and

$$
n_{y}=\frac{N_{y} L_{1}^{2}}{\sqrt{D_{11} D_{22}}}
$$

and a nondimensional pressure loading parameter is given by

$$
Q=\frac{L_{1}^{2} L_{2}^{2}}{T D} q
$$

where $q$ is the applied normal stress or internal pressure and $T$ and $D$ are given by

$$
T=\left(\frac{\sqrt{A_{11} A_{22} D_{11} D_{22}}}{A_{11} A_{22}-A_{12}^{2}}\right)^{1 / 2}
$$

and

$$
D=\sqrt{D_{11} D_{22}}
$$

Values of the nondimensional parameters for the fullscale center-body structure and the sub-scale sandwich and monolithic-laminate structural models are given in Table 3.

\section{Scaling Laws}

Scaling laws for noncircular shear-deformable, built-up composite structures subjected to combined internal pressure and mechanical loads are described in this section. The nondimensional partial differential equations given in Appendix A govern the response of a flatplate element of the full-scale structure and the sub-scale structural models, e.g., cover panels and spars. Thus, a necessary condition for complete similitude between the full-scale structure and a sub-scale structural model is that the values of the coefficients in the governing partial differential equations for each subcomponent of the subscale model must be equal to the values of the coefficients in the governing partial differential equations for the corresponding subcomponent of the full-scale structure. In addition, these equalities must be satisfied simultaneously for all subcomponents in the built-up structure. That is, the values of the nondimensional parameters given in Eqs. (1) - (10) and Eqs. (14) - (17) for 
Table 3. Nondimensional parameters

\begin{tabular}{|c|c|c|c|c|c|c|}
\hline \multirow[b]{2}{*}{ Parameter } & \multicolumn{2}{|c|}{ Full-Scale Sandwich } & \multicolumn{2}{|c|}{ 1/12-Scale Sandwich } & \multicolumn{2}{|c|}{ 1/12-Scale Laminate } \\
\hline & Cover panel & Spar & Cover panel & Spar & Cover panel & Spar \\
\hline$\mu_{l}$ & 12.26 & 0.776 & 12.26 & 0.776 & 12.26 & 0.776 \\
\hline$\mu_{2}$ & 0.317 & 0.317 & 0.317 & 0.317 & 0.317 & 0.317 \\
\hline$\mu_{3}$ & 0.599 & 0.599 & 0.599 & 0.599 & 0.599 & 0.599 \\
\hline$\alpha_{1}$ & 4.16 & 1.05 & 4.16 & 1.05 & 4.16 & 1.05 \\
\hline$\alpha_{2}$ & 0.108 & 0.427 & 0.108 & 0.427 & 0.108 & 0.427 \\
\hline$\alpha_{3}$ & 0.599 & 0.599 & 0.599 & 0.599 & 0.599 & 0.599 \\
\hline$\alpha_{4}$ & 849 & 598 & 849 & 598 & 42100 & 42400 \\
\hline$\alpha_{5}$ & 0.017 & 0.006 & 0.017 & 0.006 & 0.005 & 0.003 \\
\hline$\Gamma$ & 2.375 & 2.375 & 2.375 & 2.375 & 0.856 & 0.856 \\
\hline$\Lambda$ & 0.340 & 1.35 & 0.340 & 1.35 & 0.340 & 1.35 \\
\hline$Q$ & $193 q^{\mathrm{a}}$ & $285 q$ & $193 q$ & $285 q$ & $654 q$ & $662 q$ \\
\hline$n_{x}$ & $0.020 N_{x}^{b}$ & $0.010 N_{x}$ & $0.242 N_{x}$ & $0.125 N_{x}$ & $0.237 N_{x}$ & $0.120 N_{x}$ \\
\hline$n_{y}$ & $0.0023 N_{y}^{\mathrm{c}}$ & $0.019 N_{y}$ & $0.028 N_{y}$ & $0.227 N_{y}$ & $0.027 N_{y}$ & $0.219 N_{y}$ \\
\hline$n_{x y}$ & $0.0068 N_{x y}{ }^{d}$ & $0.014 N_{x y}$ & $0.082 N_{x y}$ & $0.168 N_{x y}$ & $0.081 N_{x y}$ & $0.162 N_{x y}$ \\
\hline$U$ & $21.76 u^{e}$ & $177.2 u$ & $216.1 u$ & $2128 u$ & $3080 u$ & $12318 u$ \\
\hline$V$ & $64.06 v^{f}$ & $131.3 v$ & $768.7 v$ & $1576 v$ & $9067 v$ & $9124 v$ \\
\hline$W$ & $0.366 w^{g}$ & $1.05 w$ & $4.40 w$ & $12.6 w$ & $15.1 w$ & $30.2 w$ \\
\hline
\end{tabular}

each subcomponent of the sub-scale structural model must be equal to the values of the corresponding parameters for the corresponding subcomponent of the fullscale structure, and these equalities must be met simultaneously for all of the subcomponents. In the context of the present study, in which the applied loads consist of an applied internal pressure load $q$ and a span-wise compression load $N_{\mathrm{x}}$, only scaling laws derived from Eqs. (1) - (10), and Eqs. (14) and (17) need to be satisfied. A second condition required for complete similitude between the full-scale structure and a sub-scale structural model is the satisfaction of the continuity conditions at the intersection of the subcomponents of the structure. More specifically, the intersection of or the joint between the individual structural elements requires that displace- ment and moment compatibility conditions between the structural elements are satisfied simultaneously. The nondimensional compatibility conditions and corresponding nondimensional parameters are derived in Appendix A. Nondimensional parameters corresponding to the displacement compatibility conditions produce the same scaling laws as Eqs. (11) - (13), and are therefore redundant. However, nondimensionalization of the moment compatibility conditions results in an additional nondimensional parameter as follows:

$$
\alpha_{6}=\frac{D_{12}}{\sqrt{D_{11} D_{22}}}
$$

The displacement response quantities $u, v$ and $w$ scale according to Eqs. (11), (12), and (13), respectively, 
and the membrane stress resultants $N_{x}, N_{y}$ and $N_{x y}$ scale according to the relations given in Eqs. (14), (15) and (16), respectively.

\section{Results and Discussion}

Analytically predicted results for three center-body structural configurations subjected to combined internal pressure and span-wise compressive loads are presented in this section. The three structural configurations include a full-scale center-body structure with a sandwich wall construction, and two sub-scale structures with a sandwich or a monolithic-laminate wall construction. The predicted results were obtained from finite-element models of each of the configurations. First, results illustrating the nonlinear response of a full-scale center-body structure subjected to combined internal pressure and span-wise compressive loads are presented. Then, predictions of the full-scale structural response based on results for the two sub-scale structural models are presented. The predictions of the full-scale structural response characteristics are made by applying the appropriate scaling laws to the sub-scale structural response results. Finally, results from an analytical parametric study illustrating the effects of partial structural similitude are presented. The results include predicted deformation response patterns, membrane stress resultant patterns, and curves indicating the effects of partial similitude on selected structural displacements.

\section{Typical Nonlinear Response of Center-body Structures}

Analytical results illustrating the nonlinear response of the full-scale and sub-scale center-body structures are presented in this section. A typical deformation pattern for the full-scale center-body structure subjected to combined internal pressure and span-wise compression loads is given in Fig. $2 \mathrm{a}$, and the corresponding pressure-displacement response curves for the center-body structure are given in Fig 2b. The center normal displacement of the upper cover panel and of the aft spar are denoted by $\delta_{1}$, and $\delta_{2}$, respectively, as shown in Fig $2 a$. The results indicate that the deformation response of the cover panel increases linearly as the pressure increases up to $15 \mathrm{psi}$, and the center normal displacement is equal to 2.1 inches for a pressure load equal to $15 \mathrm{psi}$. The span-wise compression load is then applied to the structure, and the center normal displacement of the cover panel increases to 2.7 inches. In contrast, the predicted pressure-displacement response for the spar indicates that the aft spar exhibits a nonlinear stiffening behavior when subjected to the applied pressure load. The aft spar exhibits a center displacement equal to 3.5 inches when it is subjected to a 15 psi internal pressure load. The span-wise compressive load is then applied to the structure and the center normal displacement increases to 4.4 inches. The magnitudes of the displacements in the aft spar are larger than the magnitudes of the displacements exhibited by the cover panel because of the lower bending stiffness of the spar that is associated with the particular sandwich construction of the spar. Predicted membrane stress resultant distributions for the full-scale center-body structure subjected to combined internal pressure and span-wise compression loads are shown in Fig. 3. The darker contours represent compressive stress resultants and the lighter contours represent tensile stress resultants. The results indicate that significant tensile and compressive stresses develop in the facesheets of the cover panels and the aft spar due to the large normal-displacement magnitudes. In particular, significant stress gradients exist near the boundaries of the cover panels and the aft spar.

A comparison of the full-scale structure displacement response and the predicted sub-scale structural model displacement response for the two sub-scale structural models is shown in Fig. 4. The cover-panel displacements and the aft-spar displacements are denoted by $\delta_{1}$ and $\delta_{2}$, respectively. The superscripts $f s, s$ and $m$ in the figure denote results for the full-scale structure, the sub-scale sandwich structural model, and the subscale monolithic-laminate structural model, respectively. Predictions for the full-scale structural response, and those obtained by scaling the sub-scale sandwich structural model response are shown as dashed lines in Fig. 4. Predictions for the full-scale structural response obtained by scaling the sub-scale monolithic-laminate structural model response are shown in the figure as solid lines. The results indicate that the full-scale displacement response and the sub-scale sandwich displacement response exhibit perfect similitude as evidenced by perfect agreement in the displacement response. This similitude is consistent with the fact that the sub-scale sandwich structural model satisfies all of the scaling laws simultaneously, as shown in Table 3. Additional results indicate that the membrane stress resultants also exhibit perfect similitude for the full-scale structure and the sub-scale sandwich structural model. However, the results indicate that there is a significant discrepancy between the full-scale response and the response predicted by the sub-scale monolithic-laminate structural model. The results for the sub-scale monolithic-laminate structural model and for the scaling laws under-predict the fullscale cover-panel and aft-spar displacements by approximately $40 \%$ and $31 \%$, respectively. This difference in results is due to the fact that only partial similitude exists between the sub-scale monolithic-laminate structural model and the full-scale sandwich structure. In particular, results in Table 3 indicate that there are significant differences in several of the nondimensional parameters including $\alpha_{4}, \alpha_{5}$, and $\Gamma$. The parameter $\alpha_{4}$ is a trans- 
verse shear parameter, and it is approximately two orders of magnitude larger for the sub-scale monolithic-laminate structural model than for the corresponding parameter for the sandwich structures. The parameters $\alpha_{5}$ and $\Gamma$ are also transverse shear parameters, and they are also affected in a similar manner. These results indicate that the monolithic-laminate structural model has higher transverse shear stiffness than the sandwich structural model. This higher transverse shear stiffness, in turn, causes a significant reduction in the magnitude of the normal displacements exhibited by the monolithic-laminate structural model as shown in Fig. 4.

\section{Effects of Partial Similitude}

Results from the previous section indicate that partial similitude can have a significant effect on the predicted scaled response of a built-up noncircular composite structure subjected to combined loads. Results illustrating the effects of relaxing selected similitude conditions or scaling laws on the sensitivity of the effectiveness of using the sub-scale structural model response characteristics for predicting the full-scale structural response characteristics are presented in this section. These results are determined by independently varying selected scaling laws for the models. The results are presented in terms of normalized normal displacements as a function of selected scaling parameters. Predicted full-scale cover-panel and aft-spar displacements obtained from a model that has partial similitude with the full-scale structural model are denoted by $\delta_{1}{ }^{p}$ and $\delta_{2}{ }^{p}$, respectively. These displacements are normalized with respect to the corresponding predicted full-scale coverpanel and aft-spar displacements that are denoted by $\delta_{1}$, and $\delta_{2}$, respectively. Similarly, selected nondimensional parameters with a superscript $p$ denote parameters associated with models exhibiting partial similitude, and are normalized with respect to the parameter values associated with models exhibiting perfect similitude. A normalized parametric value equal to one indicates perfect similitude for that parameter.

Results illustrating the sensitivity of the deformation response to variations in the stiffness-weighted aspect ratio $\alpha_{1}$ are shown in Fig. 5. The solid and dashed lines represent results for the structure subjected to internal pressure only and to combined internal pressure and span-wise compressive loads, respectively. The square symbols represent results for the cover panel and the triangular symbols represent results for the spar. These results indicate that the displacement responses of the cover panel and the aft spar are sensitive to variations in the parameter $\alpha_{1}$ and have normalized displacement values that range from 1.6 to 0.4 as $\alpha_{1}$ varies from 0.7 to 1.1. In addition, the results indicate that the effects of partial similitude for $\alpha_{1}$ on the response of the cover pancl can be affected by the loading conditions. For example, the magnitude of the normalized deformation response of the cover panel with $\alpha_{1}^{p} / \alpha_{1}$ equal to 0.7 and subjected to an internal pressure load only is equal to approximately 1.6 , which over-predicts the displacement by $60 \%$. However, the corresponding magnitude of the normalized deformation response of the cover panel when subjected to combined internal pressure and spanwise compressive loads equals approximately 1.35 , which over-predicts the displacements by only $35 \%$. In contrast, the results for the aft-spar structure do not vary as is a function of the loading condition. These results suggest that there is a complex nonlinear relationship between the variations in the structural similitude and the structural response.

Results illustrating the sensitivity of the deformation response to variations in the transverse shear parameter $\alpha_{4}$ are shown in Fig. 6 . The results indicate that the cover-panel deformation response can be sensitive to variations in the parameter $\alpha_{4}$. In particular, the magnitude of the normalized cover-panel displacement response decreases monotonically from approximately 2.1 to 0.6 as $\alpha_{4}$ increases. In addition, the results indicate that the effects of partial similitude of $\alpha_{4}$ on the coverpanel response can be affected by the loading conditions, but to a lesser extent than for the results for models with partial similitude in $\alpha_{1}$ as shown in Fig. 5. In contrast, the deformation response of the aft-spar structure has only a slight sensitivity to variations in $\alpha_{4}$ as indicated by the relatively benign decrease in the normalized displacement $\delta_{2}$ as $\alpha_{4}{ }^{p} / \alpha_{4}$ increases. In addition, the effects of partial similitude on the aft-spar response are insensitive to the loading conditions.

Results illustrating the sensitivity of the deformation response to variations in normalized values of the parameter $\Gamma$, which is the ratio of the transverse shear stiffnesses, are shown in Fig. 7. These results indicate that the cover-panel and aft-spar deformations are, for the most part, insensitive to variations in the ratio of the transverse shear stiffnesses. However, for a structural configuration with a shear stiffness ratio equal to 0.1 times the nominal ratio (perfect similitude) in both the cover panel and the aft spar, the cover panel has significantly larger normalized deformations than for the other values of $\Gamma$ considered. These larger deformations are the result of the buckling of the forward spar in the center-body structure. After the forward spar buckles, the internal loads are redistributed to the leading-edge structure and to the cover panels, and results in a significant increase in the magnitude of the deformations in the cover panels. 


\section{Discrepancy in the Scaling Laws}

Results comparing the predicted full-scale displacement response with the predicted full-scale displacement response determined from the two sub-scale models and the scaling laws are shown in Fig. 8. The dashed lines in the figure correspond to the predicted results from the monolithic-laminate center-body model, and the solid lines represent results from the sandwich models. The cover-panel and aft-spar displacements are denoted by $\delta_{1}$ and $\delta_{2}$, respectively. The superscripts $f s$, $s$ and $m$, denote results for the full-scale structure, subscale sandwich structure, and sub-scale monolithic-laminate structure, respectively. The two sub-scale models are constructed so that they are perfectly similar to the full-scale model according to the scaling laws presented herein. The results shown in Fig. 8 indicate that the normalized cover-panel displacement responses predicted by all three models agree well, however, the monolithiclaminate center-body model over-predicts the aft-spar deformations. This discrepancy is attributed to the fact that the sandwich spar has a greater degree of nonlinear membrane stiffening in its response, than does the monolithic-laminate spar. This nonlinear membrane stiffening effect is not accounted for in the present set of scaling laws because the governing equations of motion omit higher order bending-extension coupling terms that are necessary to account for this effect.

\section{Concluding Remarks}

Results of an analytical study of the behavior of a complex, built-up noncircular multi-cell composite structure subjected to combined internal pressure and mechanical loads are presented. In addition, the derivation of nondimensional parameters from the governing equations of motion for selected components of the builtup multi-cell structure is presented, and these nondimensional parameters are used to develop scaling laws for use with sub-scale structural models that can simulate the response of the full-scale structure. Sub-scale structural models with both complete and partial similitude are discussed. In the case of partial similitude between a subscale structural model and the full-scale structure, results from analytical parametric studies are presented, and are used to identify the sensitivity of the structural response characteristics to relaxation of similitude conditions for different scaling laws.

The results indicate that partial similitude can affect the accuracy of full-scale structural-response predictions that are predicted from sub-scale structural models, and that the sensitivity of the response to these effects can be affected by the loading conditions and by the shell-wall construction concept used for the structure. Shell-wall construction concepts with different transverse shear stiffnesses will have different results. In ad- dition, the results indicate that the accuracy of the predicted response can be affected by varying degrees of nonlinear behavior in the full-scale structure and the subscale structural models.

\section{Appendix A}

\section{Nondimensional Equations of Motion and Parameters}

The governing equations of motion for shear deformable flat plates presented herein are based on a firstorder shear-deformation plate theory given by Whitney. ${ }^{7}$ In these equations, the in-plane force effects are included by retaining the nonlinear terms in the equations of motion involving products of stresses and plate mid-surface slopes, and all other nonlinear terms are neglected. The governing equations of motion are given by

$$
\begin{gathered}
A_{11} \frac{\partial^{2} u}{\partial x^{2}}+A_{66} \frac{\partial^{2} u}{\partial y^{2}}+\left(A_{12}+A_{66}\right) \frac{\partial^{2} v}{\partial x \partial y}=0 \\
\left(A_{12}+A_{66}\right) \frac{\partial^{2} u}{\partial x \partial y}+A_{66} \frac{\partial^{2} v}{\partial x^{2}}+A_{22} \frac{\partial^{2} v}{\partial y^{2}}=0 \\
D_{11} \frac{\partial^{2} \psi_{x}}{\partial x^{2}}+D_{66} \frac{\partial^{2} \Psi_{x}}{\partial y^{2}}+\left(D_{12}+D_{66}\right) \frac{\partial^{2} \psi_{y}}{\partial x \partial y} \\
-A_{55}\left(\Psi_{x}+\frac{\partial w}{\partial x}\right)=0 \\
\left(D_{12}+D_{66}\right) \frac{\partial^{2} \Psi_{x}}{\partial x \partial y}+D_{66} \frac{\partial^{2} \Psi_{y}}{\partial x^{2}}+D_{22} \frac{\partial^{2} \Psi_{y}}{\partial y^{2}} \\
-A_{44}\left(\Psi_{y}+\frac{\partial w}{\partial y}\right)=0 \\
A_{55}\left(\frac{\partial \psi_{x}}{\partial x}+\frac{\partial^{2} w}{\partial x^{2}}\right)+A_{44}\left(\frac{\partial \psi_{y}}{\partial y}+\frac{\partial^{2} w}{\partial y^{2}}\right)+N_{x} \frac{\partial^{2} w}{\partial x^{2}} \\
+N_{x y} \frac{\partial^{2} w}{\partial x \partial y}+N_{y} \frac{\partial^{2} w}{\partial y^{2}}+q=0
\end{gathered}
$$

where $A_{11}, A_{12}, A_{22}$ and $A_{66}$ are the shell membrane stiffnesses and $D_{11}, D_{12}, D_{22}$ and $D_{66}$ are the shell bending stiffnesses from classical laminated plate theory. The quantities $A_{44}$ and $A_{55}$ are the effective transverse shear stiffnesses and are equal to $G_{23} t$ and $G_{13} t$, respectively, where $t$ is the plate thickness. The quantities $u$ and $v$ are the in-plane displacements of the plate and $w$ is the normal deflection of the plate. The quantities $\psi_{\mathrm{x}}$ and $\psi_{\mathrm{y}}$ represent the total rotation of the normal to the mid-surface of the plate. The quantities $N_{x}$, $N_{y}$ and $N_{x y}$ are the membrane stress resultants.

Following Stein ${ }^{5}$ and Nemeth, ${ }^{6}$ the nondimensional coordinates 


$$
S_{1}=x / L_{1} \text { and } S_{2}=y / L_{2}
$$

are used, where $L_{1}$ and $L_{2}$ are characteristic dimensions of the of the built-up plate structure shown in Fig. 1. Substituting these coordinates into Eq. (Al) and normalizing by the geometric mean in-plane stiffness; i.e., multiplying through by $L_{1} L_{2} / \sqrt{A_{11} A_{22}}$ yields

$$
\mu_{1} \frac{\partial^{2} U}{\partial S_{1}^{2}}+\mu_{2} \frac{\partial^{2} U}{\partial S_{2}^{2}}+\mu_{3} \frac{\partial^{2} V}{\partial S_{1} \partial S_{2}}=0
$$

where the nondimensional parameters appearing in the equation are given by

$$
\begin{gathered}
\mu_{1}=\left(\frac{L_{2}}{L_{1}}\right)^{2} \sqrt{\frac{A_{11}}{A_{22}}} \\
\mu_{2}=\frac{A_{66}}{\sqrt{A_{11} A_{22}}} \\
\mu_{3}=\frac{A_{12}+A_{66}}{\sqrt{A_{11} A_{22}}}
\end{gathered}
$$

and nondimensional in-plane displacements are given by

$$
U=\frac{A_{11} A_{22}-A_{12}^{2}}{\sqrt{A_{11} A_{22} D_{11} D_{22}}} L_{1} u
$$

and

$$
V=\frac{A_{11} A_{22}-A_{12}^{2}}{\sqrt{A_{11} A_{22} D_{11} D_{22}}} L_{2} v
$$

Similarly Eq. (A2) becomes

$$
\mu_{3} \frac{\partial^{2} U}{\partial S_{1} \partial S_{2}}+\frac{1}{\Lambda^{2}} \mu_{2} \frac{\partial^{2} V}{\partial S_{1}^{2}}+\mu_{1}^{-1} \frac{\partial^{2} V}{\partial S_{2}^{2}}=0
$$

where $\Lambda=L_{1} / L_{2}$. Proceeding in a similar manner, and substituting the nondimensional coordinates into Eq. (A3) and normalizing by the geometric mean bending stiffness; i.e., multiplying through by $L_{1} L_{2} / \sqrt{D_{11} D_{22}}$ yields

$$
\begin{aligned}
& \frac{L_{2}}{L_{1}} \sqrt{\frac{D_{11}}{D_{22}}} \frac{\partial^{2} \Psi_{x}}{\partial S_{1}^{2}}+\frac{L_{1}}{L_{2}} \frac{D_{66}}{\sqrt{D_{11} D_{22}}} \frac{\partial^{2} \Psi_{x}}{\partial S_{2}^{2}} \\
& +\frac{D_{12}+D_{66}}{\sqrt{D_{11} D_{22}}} \frac{\partial^{2} \Psi_{y}}{\partial S_{1} S_{2}} \\
& -\frac{L_{1} L_{2} A_{55}}{\sqrt{D_{11} D_{22}}}\left(\psi_{x}+\frac{1}{L_{1}} \frac{\partial w}{\partial S_{1}}\right)=0
\end{aligned}
$$

Let

$$
W=\left(\frac{A_{11} A_{22}-A_{12}^{2}}{\sqrt{A_{11} A_{22} D_{11} D_{22}}}\right)^{1 / 2}
$$

then, Eq. (A13) becomes

$$
\begin{aligned}
& \alpha_{1} \frac{\partial^{2} \psi_{x}}{\partial S_{1}^{2}}+\alpha_{2} \frac{\partial^{2} \psi_{x}}{\partial S_{2}^{2}}+\alpha_{3} \frac{\partial^{2} \psi_{y}}{\partial S_{1} \partial S_{2}} \\
& -\alpha_{4}\left(\psi_{x}+\alpha_{5} \frac{\partial W}{\partial S_{1}}\right)=0
\end{aligned}
$$

where the nondimensional parameters appearing in the equation are given by

$$
\begin{aligned}
& \alpha_{1}=\frac{L_{2}}{L_{1}} \sqrt{\frac{D_{11}}{D_{22}}} \\
& \alpha_{2}=\frac{L_{1}}{L_{2}} \frac{D_{66}}{\sqrt{D_{11} D_{22}}} \\
& \alpha_{3}=\frac{D_{12}+D_{66}}{\sqrt{D_{11} D_{22}}} \\
& \alpha_{4}=\frac{L_{1} L_{2} A_{55}}{\sqrt{D_{11} D_{22}}}
\end{aligned}
$$

and

$$
\alpha_{5}=\frac{1}{L_{1}}\left(\frac{\sqrt{A_{11} A_{22} D_{11} D_{22}}}{A_{11} A_{22}-A_{12}^{2}}\right)^{1 / 2}
$$

Similarly, Eq. (A4) becomes

$$
\begin{aligned}
& \beta_{1} \frac{\partial^{2} \Psi_{x}}{\partial S_{1} \partial S_{2}}+\beta_{2} \frac{\partial^{2} \psi_{y}}{\partial S_{1}^{2}}+\beta_{3} \frac{\partial^{2} \psi_{y}}{\partial S_{2}^{2}} \\
& -\beta_{4}\left(\psi_{y}+\beta_{5} \frac{\partial W}{\partial S_{2}}\right)=0
\end{aligned}
$$

where the nondimensional parameters appearing in the equation are given by

$$
\begin{aligned}
& \beta_{1}=\alpha_{3} \\
& \beta_{2}=\frac{1}{\Lambda^{2}} \alpha_{2} \\
& \beta_{3}=\alpha_{1}^{-1} \\
& \beta_{4}=\Gamma \alpha_{4} \\
& \beta_{5}=\Lambda \alpha_{5}
\end{aligned}
$$

where $\Gamma=A_{44} / A_{55}$. Similarly Eq. (A5) becomes 


$$
\begin{aligned}
& \frac{\alpha_{4}}{L_{1}}\left(\frac{\partial \psi_{x}}{\partial S_{1}}+\alpha_{5} \frac{\partial^{2} W}{\partial S_{1}^{2}}\right)+\frac{\beta_{4}}{L_{2}}\left(\frac{\partial \psi_{y}}{\partial S_{2}}+\beta_{5} \frac{\partial^{2} W}{\partial S_{2}^{2}}\right) \\
& +\frac{\alpha_{5}}{L_{2}}\left(n_{x} \frac{\partial^{2} W}{\partial S_{1}^{2}}+n_{x y} \frac{\partial^{2} W}{\partial S_{1} \partial S_{2}}+n_{y} \frac{\partial^{2} W}{\partial S_{2}^{2}}\right) \\
& +\frac{L_{1} L_{2}}{\sqrt{D_{11} D_{22}}} q=0
\end{aligned}
$$

where the nondimensional parameters appearing in the equation are defined by

$$
\begin{aligned}
& n_{x}=\frac{N_{x} L_{2}^{2}}{\sqrt{D_{11} D_{22}}} \\
& n_{x y}=\frac{N_{x y} L_{1} L_{2}}{\sqrt{D_{11} D_{22}}}
\end{aligned}
$$

and

$$
n_{y}=\frac{N_{y} L_{1}^{2}}{\sqrt{D_{11} D_{22}}}
$$

Multiplying Eq. (A28) through by $L_{2} / \alpha_{5}$ yields

$$
\begin{aligned}
& \frac{1}{\Lambda} \alpha_{4}\left(\frac{1}{\alpha_{5}} \frac{\partial \psi_{x}}{\partial S_{1}}+\frac{\partial^{2} W}{\partial S_{1}^{2}}\right) \\
& +\Gamma \alpha_{4}\left(\frac{1}{\alpha_{5}} \frac{\partial \psi_{y}}{\partial S_{2}}+\Lambda \frac{\partial^{2} W}{\partial S_{2}^{2}}\right) \\
& +n_{x} \frac{\partial^{2} W}{\partial S_{1}^{2}}+n_{x y} \frac{\partial^{2} W}{\partial S_{1} \partial S_{2}}+n_{y} \frac{\partial^{2} W}{\partial S_{2}^{2}}+Q=0
\end{aligned}
$$

where

$$
\begin{gathered}
Q=\frac{L_{1}^{2} L_{2}^{2}}{T D} q \\
T=\left(\frac{\sqrt{A_{11} A_{22} D_{11} D_{22}}}{A_{11} A_{22}-A_{12}^{2}}\right)^{1 / 2}
\end{gathered}
$$

and

$$
D=\sqrt{D_{11} D_{22}}
$$

\section{Typical Nondimensional Compatibility Conditions}

Typical displacement compatibility conditions at the intersection of or the joint between two substructure elements, e.g.; the aft spar and the top cover panel, are as follows:

$$
\begin{aligned}
& v_{c p}=w_{s p u r} \\
& w_{c p}=v_{\text {spur }}
\end{aligned}
$$

$$
u_{c p}=u_{x p a r}
$$

where the subscripts $c p$ and spar indicate response quantities for the cover panel and the spar, respectively. Nondimensional versions of these displacement compatibility equations follow from using the appropriate nondimensional displacements given by Eqs. (Al1), (A12), and (A15). The moment compatibility condition at the intersection of the cover panel and the spar is given by

$$
M_{y, c p}=M_{y, s p a r}
$$

where

$$
M_{y}=D_{12} \frac{\partial \psi_{x}}{\partial S_{1}}+D_{22} \frac{\partial \psi_{y}}{\partial S_{2}}
$$

By following a similar nondimesionalization procedure, one obtains the nondimensional form of the moment $M_{y}$ given by

$$
m_{y}=\Lambda \alpha_{6} \frac{\partial \psi_{x}}{\partial S_{1}}+\alpha_{1}^{-1} \frac{\partial \psi_{y}}{\partial S_{2}}
$$

where $\alpha_{6}$ is given by

$$
\alpha_{6}=\frac{D_{12}}{\sqrt{D_{11} D_{22}}}
$$

\section{References}

1. Simitses, G. J., and Rezaeepazhand, J., "Structural Similitude for Laminated Structures," Journal of Composites Engineering, Vol. 3, 1993, pp. 751-765.

2. Rezaeepazhand, J., Simitses, G. J., and Starnes, J. H., Jr., "Design of Scaled Down Models for Predicting Shell Vibration Response," Journal of Sound and Vibration, Vol. 195, 1996, pp. 301-311.

3. Rezaeepazhand, J., Simitses, G. J., and Starnes, J. H., Jr., "Scale Models for Laminated Cylindrical Shells Subjected to Axial Compression," Composite Structures, Vol. 34, 1996, pp. 371-379.

4. Brogan, F. A., Rankin, C. C., Loden, W. A., and Cabiness, H. D., "STAGS User Manual," Lockheed Martin Missiles \& Space Co., Inc., Advanced Technology Center, Palo Alto, CA, Report LMSC P032594, 1999.

5. Stein, M. "Postbuckling of Orthotropic Composite Plates Loaded in Compression," AIAA Journal, Vol. 21, December, 1983.

6. Nemeth, M. P., "Nondimensional Parameters and Equations for Buckling of Symmetrically Laminated Thin Elastic Shallow Shells," NASA Technical Memorandum 104060, March, 1991.

7. Whitney, J. M., Structural Analysis of Laminated Anisotropic Plates, Technomic Publishing Co., Inc., Landcaster, PA, 1987. 


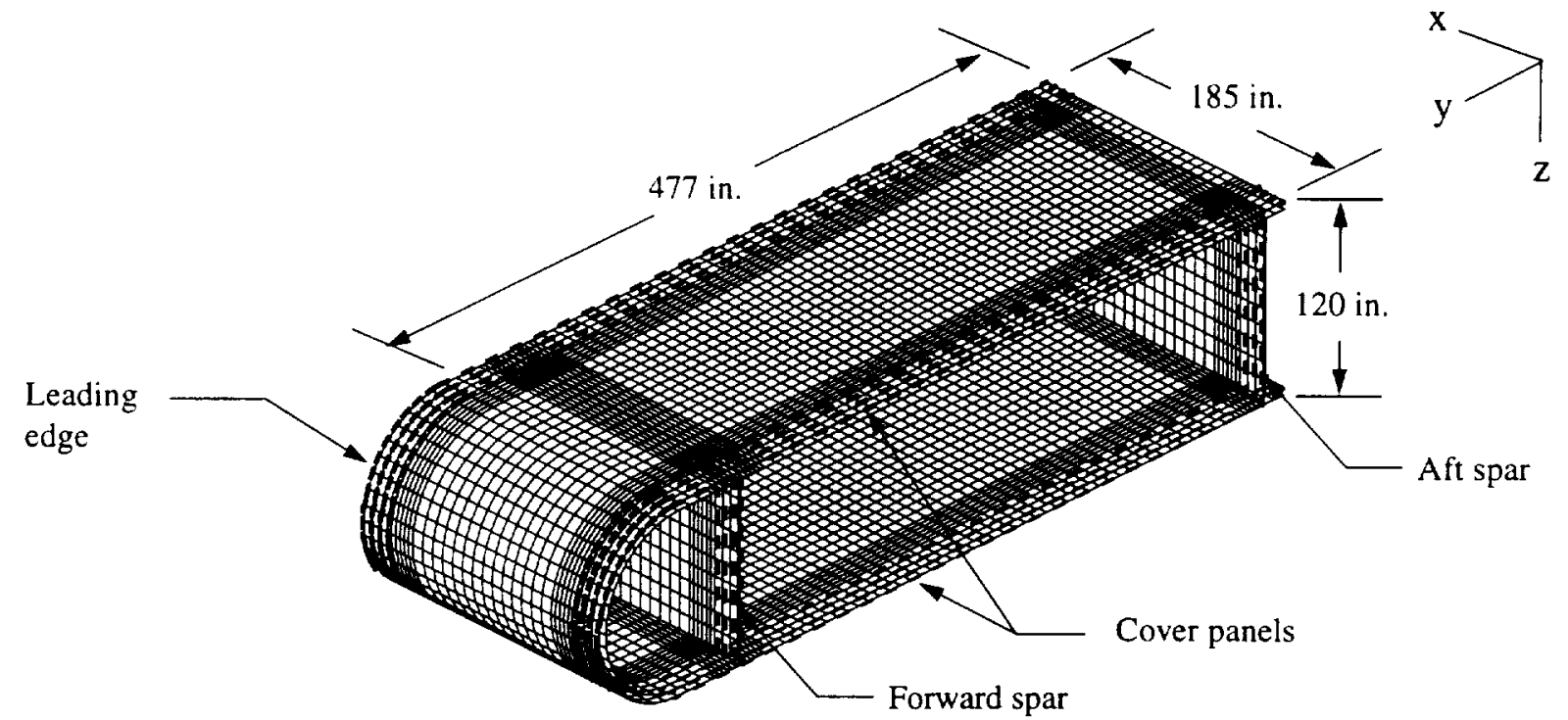

Fig. 1. Center-body structural configuration. The $x$ and $y$ coordinates correspond to the span-wise and chord-wise coordinates, respectively (dashed lines mark the rows on which the boundary conditions are applied).

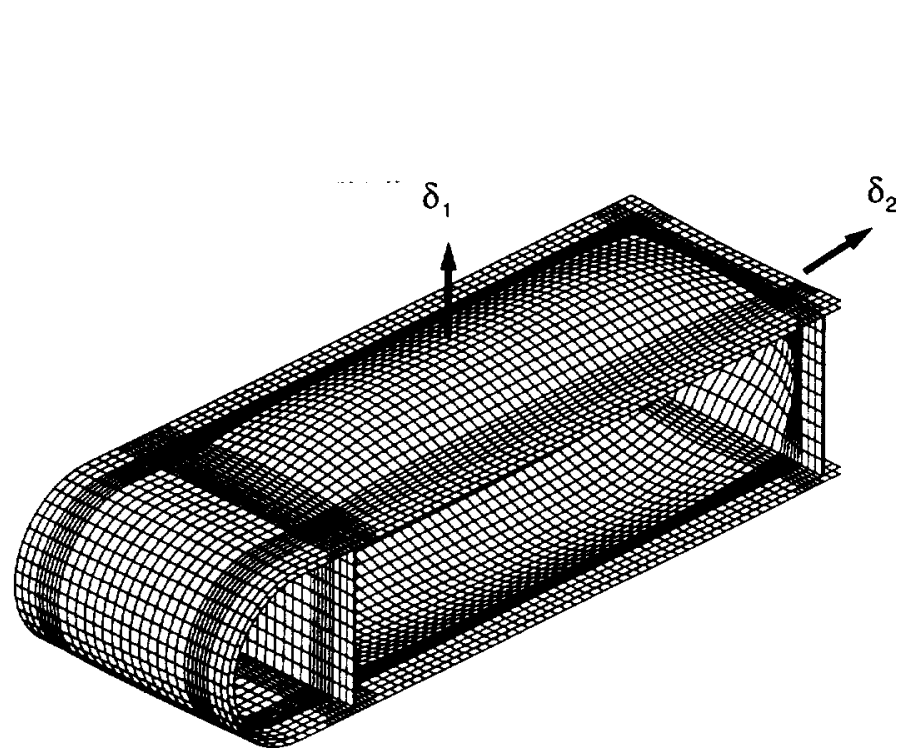

a) Deformation response

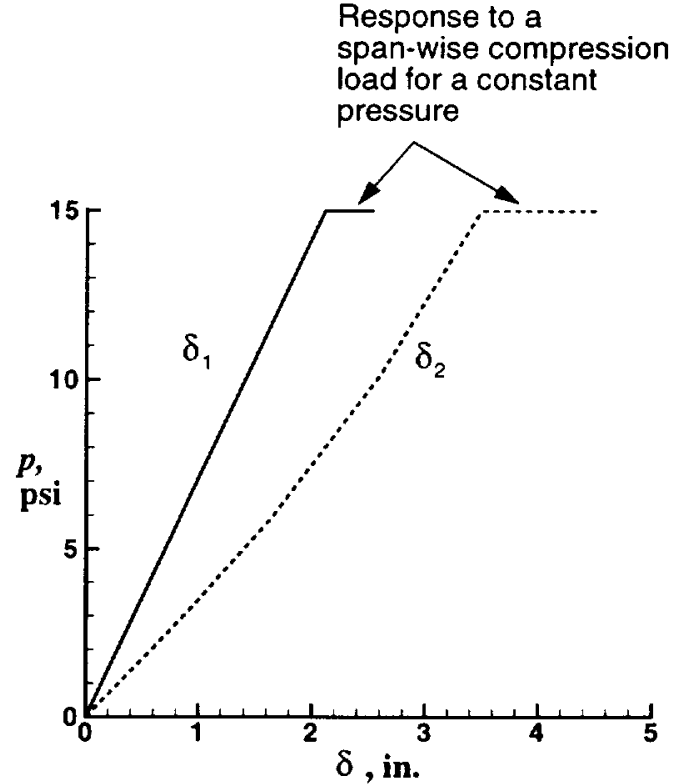

b) Pressure-displacement response curves

Fig. 2. Predicted displacement response of a full-scale center-body structure subjected to combined internal pressure and span-wise compressive loads. 

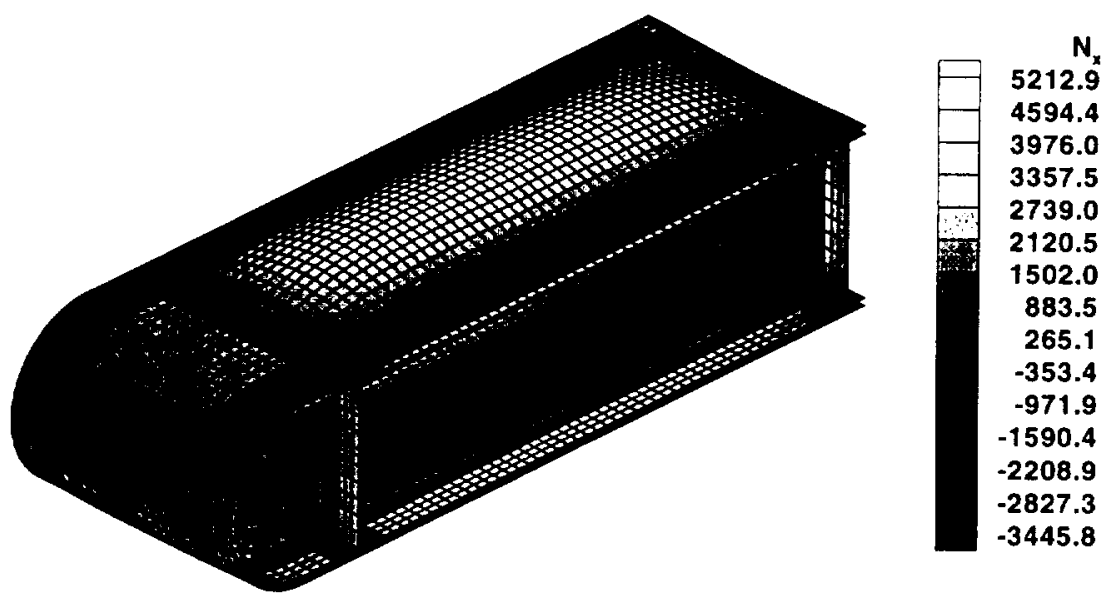

a) Span-wise stress resultant contours, $N_{x}, l b f / i n$.

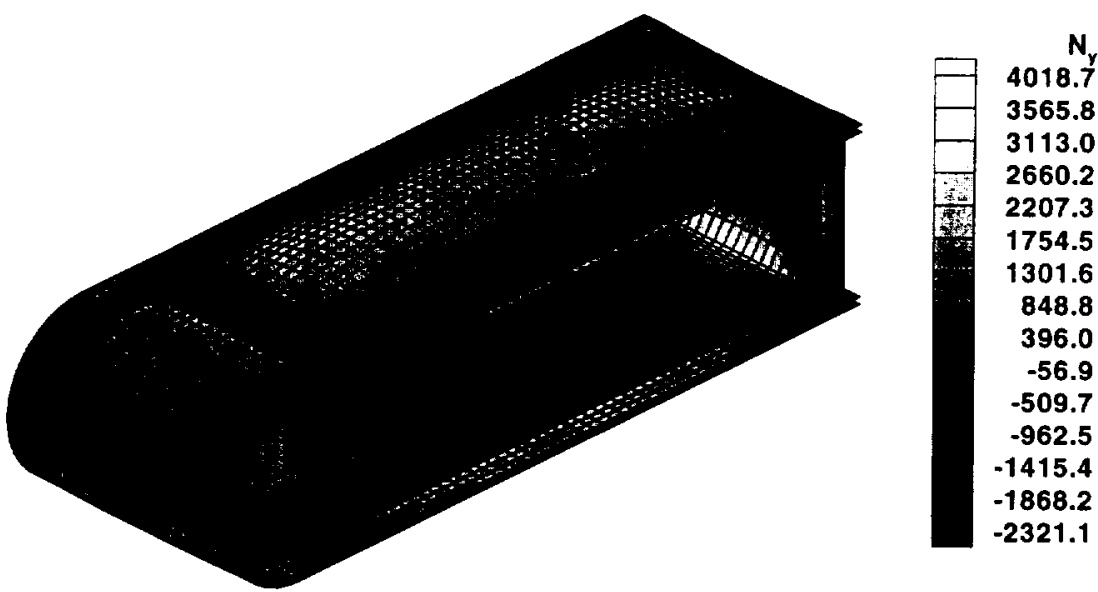

b) Chord-wise stress resultant contours, $\mathrm{N}_{\mathrm{y}}, \mathrm{lbf} / \mathrm{in}$.

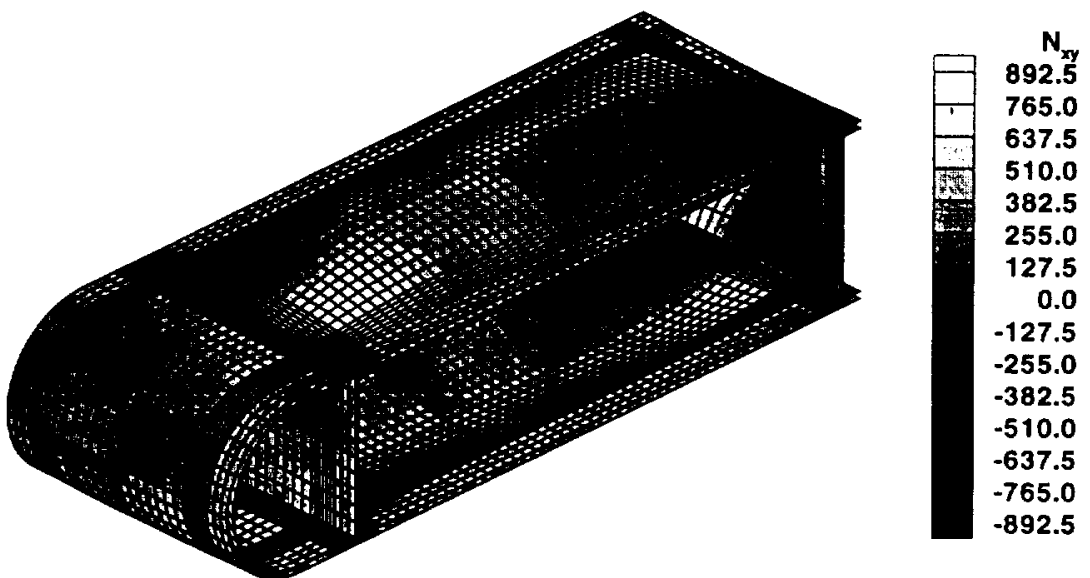

c) Shear stress resultant contours, $N_{x y}$, lbf/in.

Fig. 3. Predicted stress resultants of a full-scale center-body structure subjected to combined internal pressure and span-wise compressive loads. 


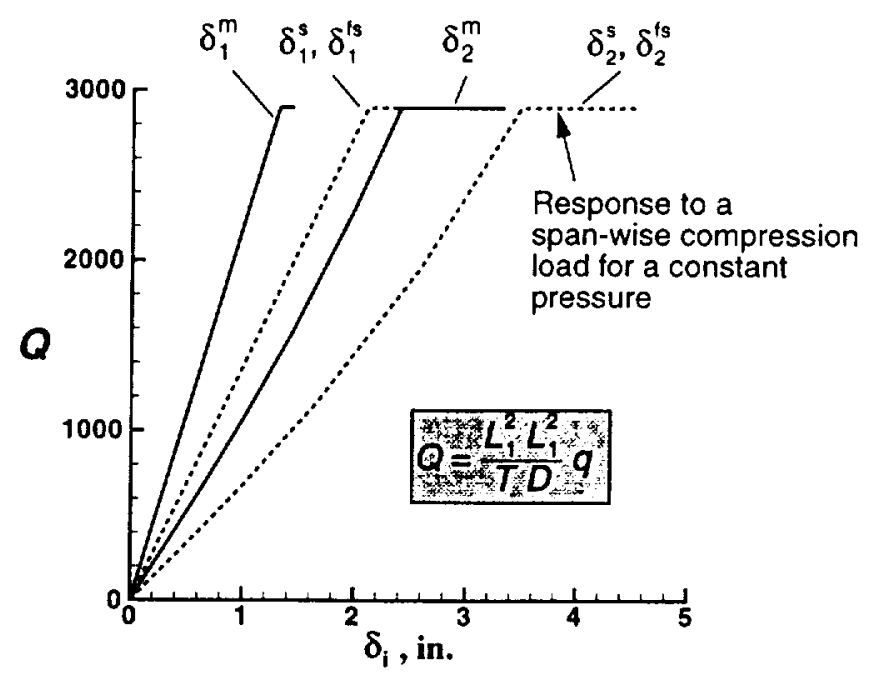

Fig. 4. Comparison of full-scale response with predicted response obtained from sub-scale models of centerbody structures subjected to combined internal pressure and span-wise compressive loads. Superscripts $f s, m$, and $s$ denote full-scale structure, sub-scale sandwich structure, and sub-scale monolithic laminate structure, respectively.

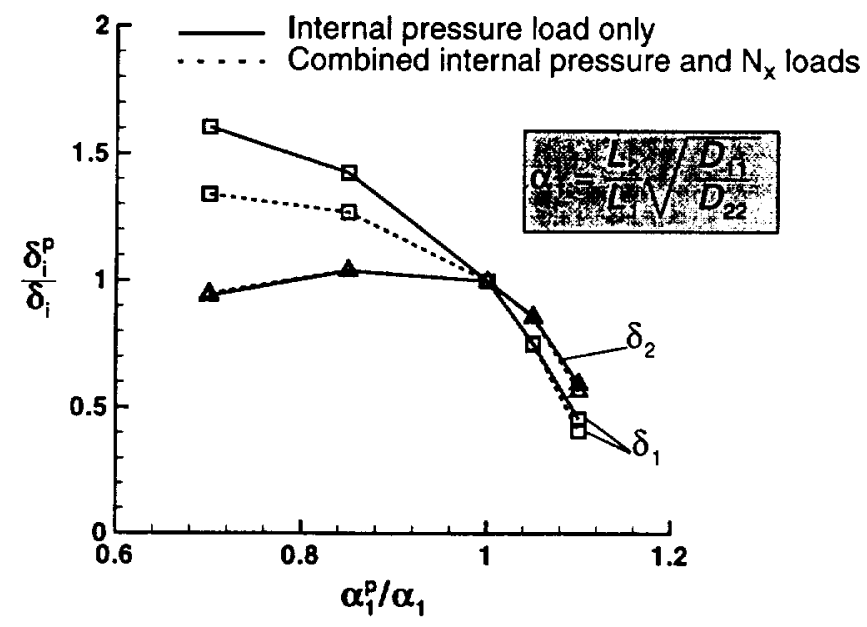

Fig. 5. Effect of variation in the $\alpha_{1}$ parameter on the deformation response of sub-scale center-body structure subjected to internal pressure only and to combined internal pressure and span-wise compressive loads. Superscript $p$ denotes parameter values and results of models with partial similitude. 


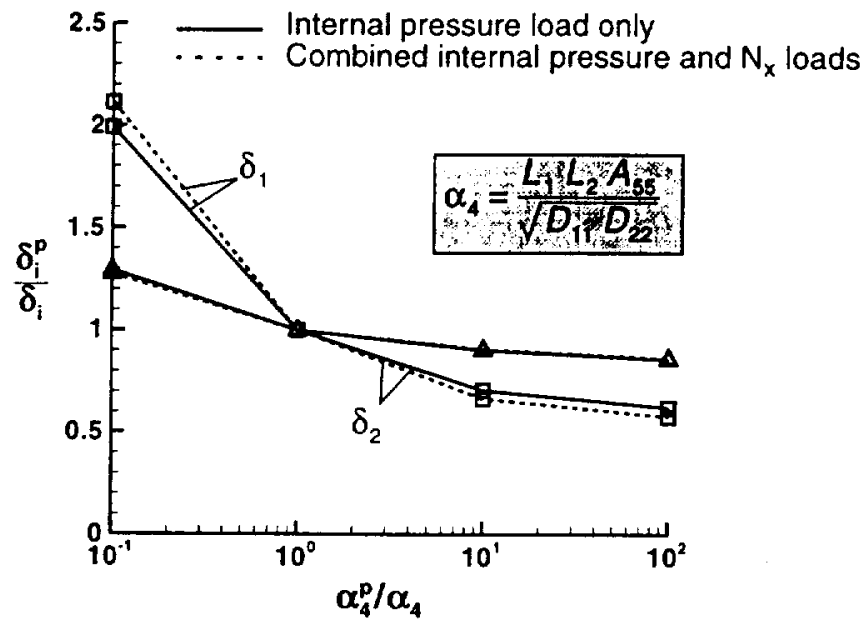

Fig. 6. Effect of variation in the $\alpha_{4}$ parameter on the deformation response of center-body structure subjected to internal pressure only and to combined internal pressure and span-wise compressive loads. Superscript $p$ denotes parameter values and results of models with partial similitude.

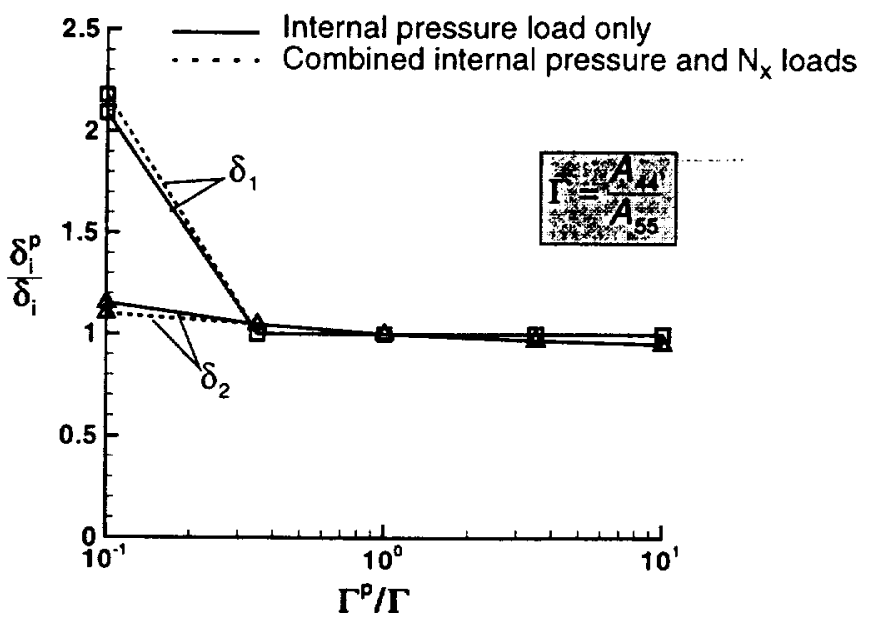

Fig. 7. Effect of variation in the $\Gamma$ parameter on the deformation response of center-body structure subjected to internal pressure only and to combined internal pressure and span-wise compressive loads. Superscript $p$ denotes parameter values and results of models with partial similitude. 


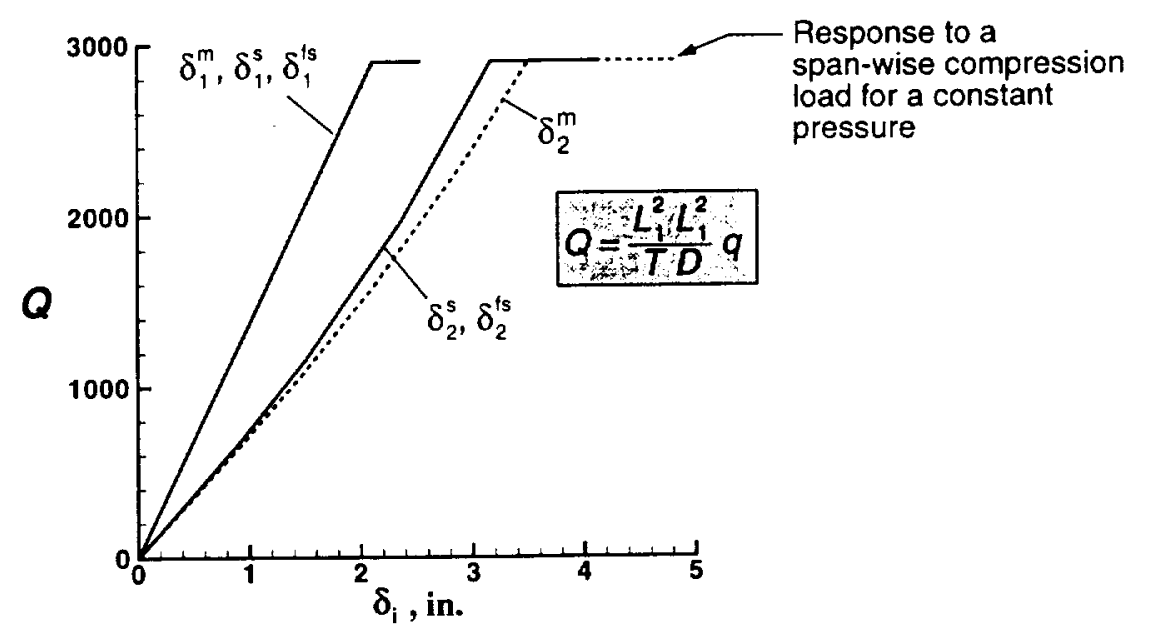

Fig. 8. Predicted displacement response of selected sub-scale center-body structures with perfect structural similitude subjected to combined internal pressure and span-wise compressive loads. Superscripts $m$ and $s$ denote monolithic and sandwich construction, respectively. 